\title{
La corruption dans les « libelles d'Estat » durant la période de la Ligue (1584-1598)
}

\section{Martial Martin}

\section{(2) OpenEdition \\ 1 Journals}

Édition électronique

URL : http://journals.openedition.org/edl/901

DOI : 10.4000/edl. 901

ISSN : 2296-5084

Éditeur

Université de Lausanne

\section{Édition imprimée}

Date de publication : 15 décembre 2015

ISBN : 978-2-940331-47-5

ISSN : 0014-2026

\section{Référence électronique}

Martial Martin, «La corruption dans les « libelles d'Estat » durant la période de la Ligue (1584-1598) », Études de lettres [En ligne], 3-4 | 2015, mis en ligne le 01 décembre 2018, consulté le 15 décembre 2020. URL : http://journals.openedition.org/edl/901 ; DOI : https://doi.org/10.4000/edl.901

\section{(C) Études de lettres}




\section{LA CORRUPTION DANS LES «LIBELLES D’ESTAT» DURANT LA PÉRIODE DE LA LIGUE (1584-1598)}

C'est contre ("en opposition à», mais aussi "en appui sur») une pensée théologique de la corruption largement diffusée par les polémiques religieuses précédentes que s'articule un discours politique en voie d'autonomisation dans les libelles de la seconde partie du règne d'Henri III et du début de celui d'Henri IV. La reprise de la critique de la corruption dans ces "pamphlets" rend d'abord compte de la difficulté du positionnement de la Ligue à ses origines (l'une des deux versions du manifeste du mouvement privilégiant la dimension politico-financière de la corruption, l'autre tentant de se raccorder à une tradition religieuse); cependant, ensuite, autour de 1588, dans un contexte où les favoris du roi Henri III, et singulièrement Epernon, cristallisent les accusations d'accaparement des biens royaux et de sorcellerie, la corruption devient un thème unificateur qui explique le succès de la propagande ligueuse contre une défense royale qui ne parvient pas à retourner ces imputations contre les leaders charismatiques de la Ligue; enfin, ce sont les dissensions internes au mouvement qui débouchent, après 1592, sur des accusations d'ambition personnelle ou de subornation aux intérêts étrangers contre les nobles et sur la dénonciation des turpitudes de la populace; tout en les vidant de leur dimension religieuse, la nouvelle propagande royale organisée autour d'Henri IV récupère ces discours ligueurs en reconnaissant en eux la cause première des conflits armés, des misères de la guerre et de la corruption du royaume de France. Le libelle des $\mathrm{XVI}^{\mathrm{e}}$ et $\mathrm{XVII}{ }^{\mathrm{e}}$ siècles se reconnaît ainsi au centre de la réflexion sur les pouvoirs de la langue: s'il prétend rétablir l'ordre en dénonçant la corruption, il se révèle souvent luimême corrupteur.

C'est autour de l'acceptation théologique de la notion de corruption que se met en place, très tôt, dans le cadre des polémiques religieuses, en amont même des troubles civils, une rhétorique du blâme duale qui, d'un côté, reprend les mœurs des hommes d'Eglise qui pervertissent le dogme et qui, de l'autre, flétrit l'hérétique qui pollue tout ce qu'il approche. Si, dans le premier cas de figure, l'on réactive souvent une satire anticléricale 
traditionnelle, dans l'autre l'on se rattache plutôt au genre de l'invective prophétique: celle-ci met en particulier en cause les pouvoirs judiciaire et politique pour le rôle qu'ils joueraient dans la corruption d'un monde devenu le négatif de ce pour quoi Dieu l'avait créé ${ }^{1}$ et à même de se rétablir seulement grâce à la puissance de la Vérité, c'est-à-dire dans la parole du prophète qui annonce la parousie ${ }^{2}$. C'est à cette parole déjà établie que s'articule, durant la seconde partie du règne d'Henri III et au début de celui d'Henri IV, le discours politique de la corruption dans les «libelles d'Estat" pour désigner avec Pierre de L'Estoile, ces brochures, de taille variable mais souvent modeste, "taxans l'impudicité du Siecle, la Bombance des Mignons, les Corruptions et Desbordemens de la Cour, l'abus des Finances et les larrecins des Financiers, les Voleries des Gouverneurs, les Vices et Paillardises des Prestres et Ecclesiastiques, l'impunité des Massacres et Massacreurs, la plumerie de l'Italie. Et en general tous les maux d'un Estat bien malade tel qu'estait celui de la France» (ajout de 1577 au Registre-Journal). Et c'est à la suite de cette tradition polémique religieuse que se comprend particulièrement la production "pamphlétaire" qui doit, en un premier temps, retenir notre attention, celle de la Ligue-même, ce mouvement catholique intransigeant animé par les Guise et leurs partisans et appuyé sur une population hostile à la succession d'un prince protestant au trône de France, devenue plus probable, après 1584 , faute d'héritier pour le couple royal, avec la mort du dernier fils de Catherine de Médicis, François d'Anjou ${ }^{3}$. Cependant, l'on assiste aussi à une forme d'autonomisation progressive et mesurée, néanmoins réelle, de l'idée de corruption politique; le manifeste de Péronne, acte de naissance de la Ligue, rend bien compte de cette ambiguïté. En fait, deux manifestes furent successivement signés à Péronne (Picardie), l'un le 5 juin 1576, l'autre le 31 mars 1585:

Le choix de Péronne [pour la seconde fois] marquait la volonté de prouver la filiation entre les mouvements de 1576 et 1585 . C'était non seulement une façon de remonter aux sources, mais surtout de se rattacher à cette ligue qu’avait créée le roi Henri III. Par là, le duc de Guise voulait prouver que l'organisation nouvelle était dans la continuité de

I. On reconnaîtra la matrice de ce discours dans Ezéchiel 28, autour de l'orgueil puni du prince de Tyr comme image de la chute.

2. Voir D. Crouzet, Les guerriers de Dieu.

3. Voir J.-M. Constant, La Ligue. 
l'ancienne et qu'il était animé par le seul souci de défendre la religion catholique face aux protestants. Les historiens [...] ont été abusés par cette propagande guisarde et sont tombés dans le piège tendu par le Balafré. En réalité, il faut bien constater qu'en 1585 la noblesse picarde était étrangement calme ${ }^{4}$.

En 1576, en effet, «Jacques d'Humières, gouverneur de Péronne, ne veut pas remettre cette ville entre les mains de Condé. Ce dernier refus a un retentissement considérable; la Picardie est en effet une province dont la position stratégique en fait un enjeu important; elle est surveillée de près par les Espagnols depuis les Pays-Bas tout proches, car ils ne tiennent pas à voir s'y installer Condé. Des seigneurs voisins de Péronne, en particulier Michel d'Estourmel et Jacques d'Happlaincourt, s'accordent le 5 juin sur un texte hardi, signé ensuite par deux cents personnes ${ }^{5}$ "; ce texte a été publié en 1576 sous un titre hostile par les adversaires des ligués: Conspiration faicte en Picardie, sous fausses et meschantes calomnies, contre l'édit de pacification.

Le contenu de la déclaration du 31 mars $1585^{6}$ n'introduit qu'une dimension relativement plus religieuse au conflit; voici comment JeanMarie Constant la résume ${ }^{7}$ :

Certains de la reprise imminente de la guerre civile, les princes responsables de la Ligue, qui avaient inspiré ce texte, engageaient les catholiques à s'y préparer parce qu'ils considéraient que des "complices» des hérétiques "glissés dans l'amitié du roi» travaillaient à l'arrivée d'un souverain huguenot. Ces mots visaient les ducs de Joyeuse et d'Espernon, mais les noms des deux favoris n'étaient pas cités. Cependant, on les accusait «d'accaparer l'Etat» de «dépouiller les uns des titres de leur dignité, les autres du pouvoir de leur fonction", de forcer les titulaires de charges à les abandonner afin de se «rendre maitres des armes par terre et par mer» (p. 9-10 ${ }^{8}$ ).

4. Ibid., p. 122 sq.

5. A. Jouanna, J. Boucher, D. Biloghi, Histoire et dictionnaire des guerres de religion, p. 242.

6. Declaration des causes qui ont meu Monseigneur le Cardinal de Bourbon et les Pairs, Princes, Seigneurs, Villes et Communautez catholiques de ce Royaume de s'opposer par armes à ceux qui veulent subvertir la Religion.

7. J.-M. Constant, La Ligue, p. 123-125.

8. C'est nous qui précisons les pages dans l'édition Basson. 
[...] Un autre thème, plus révolutionnaire, se trouve dans le texte: la réunion des états généraux tous les trois ans (p. 16). Cette idée avait sans doute été inspirée par l'aile radicale et parisienne de la Ligue, tout comme les propositions économiques et sociales: la suppression des tailles, des aides et subsides extraordinaires établis depuis la mort de Charles IX, ce qui était une façon de souligner le rôle néfaste d'Henri III dans l'alourdissement de la fiscalité (p. 15).

[...] Quoique la question dynastique et le maintien du culte romain tinssent une large place dans ce programme (p. 5, 12), tous les observateurs ne pouvaient qu'être frappés par l'insistance mise sur la responsabilité des favoris pour expliquer les désordres de l'Etat et de l'Eglise.

Il s'agit donc d'abord de lutter contre la corruption du pouvoir royal par les mignons du roi, qui en s'accaparant ses faveurs dénaturent gravement le système féodal traditionnel. Sous leur influence, la royauté pourrait tourner en tyrannie. La Ligue se destinerait d'abord à restaurer la forme première de la monarchie telle qu'elle s'exprimait encore sous Henri II. Le manifeste participe par là d'une réflexion plus ample qui, d'après Nicolas Le Roux, cherche à "comprendre les causes de la corruption des pratiques politiques qui visaient à associer la noblesse à l'exercice du pouvoir. Cette interprétation politique des troubles repose sur l'idée centrale de la perversion du modèle familial et économique du gouvernement fondé sur la répartition équilibrée des honneurs et des faveurs» ${ }^{9}$.

Cependant, les critiques ne tardèrent guère, vis-à-vis d'un mouvement qui se donnait comme continuateur de la défense de la religion mais qui n'y faisait que peu référence; aussi une seconde version de la Declaration datée du 20 avril ${ }^{10}$ tente de remédier aux maladresses du premier manifeste; elle gomme toute référence aux favoris, n'évoque qu'accessoirement les impôts nouveaux, ne laisse par contre de déplorer la ruine de la religion, appelle le roi de Navarre et le prince de Condé à abjurer, peint dans le détail les terribles conséquences de l'avènement d'un prince de «la nouvelle opinion» au trône de France, et se trouve

9. N. Le Roux, La faveur du roi, p. 21.

Io. Declaration des causes qui ont meu Monseigneur le Cardinal de Bourbon et les Pairs, Princes, Prelats, Seigneurs, Villes et Communautez catholiques de ce Royaume de s'opposer par armes à ceux qui veulent subvertir la Religion. 
dans l'obligation de réfuter toute dimension critique vis-à-vis de l'actuel pouvoir monarchique:

[...] à fin que personne ne puisse douter de nostre droite et saincte intention, et que toute occasion soit ostee à ceux qui nous ont mal affectionnez de calomnier nos actions et publier (comme ils font desja pour rendre la cause de la prinse des armes odieuse et injuste) que c'est contre l'estat et le Roy (la personne duquel nous honnorons comme l'ymage du Dieu vivant) lesdicts Princes et Seigneurs catholiques pour purger ceste calomnie et soupçon, offrent et sont prests de mettre es mains de sa dicte Majesté ce qu'ils ont de plus cher, leurs femmes et leurs enfans... ${ }^{11}$.

Trois ans plus tard, en mai 1588, après le soulèvement des ligueurs parisiens contre Henri III et l'exil forcé du roi hors de sa capitale, les tentatives de conciliation entre le monarque et les puissants Guise passent par l'éloignement du favori Epernon, opposant acharné des princes lorrains et, pour eux, cause de la corruption du pouvoir; celuici est la cible d'une véritable campagne de désinformation inaugurée par des libelles qui jouent sur un parallèle entre le duc et Gaverston (ou Gaveston), le favori du roi Edouard II d'Angleterre (début du XIV siècle), dans la tradition d'une lecture typologique de l'Histoire, où le passé préfigure le présent: L'Estrange amitié d'Edouard Second, Roy d'Angleterre à l'endroit de Pierre de Gaverston, Gentilhomme de Gascogne, et quelle en fut l'yssue, reprise des chroniques tout juste accommodée pour évoquer suffisamment le contexte du règne d'Henri III, puis l'Histoire tragique et memorable de Pierre de Gaverston Gentil-homme gascon jadis mignon d'Edoüard 2. Roy d'Angleterre tirée des Chroniques de Thomas Walsingham et tournée du Latin en François Dédiée à Monseigneur le Duc d'Espernon, identique au précédent titre à ceci près qu'elle est accompagnée d'une anagramme Pierre de Gaverston/Perjure de Nogarets (expliquant le choix orthographique sur le nom du favori anglais), d'un quatrain, d'une épître dédicatoire et d'un sonnet "Au Roy» développant le double parallèle Epernon/Gaveston, Henri III/Edouard II. Le mignon fait rédiger en réponse un Antigaverston qui n'a pas été conservé; les rebelles contre-attaquent rapidement avec les Replique à l'antigaverston, ou responce faicte à l'histoire de Gaverston par le duc d'Espernon et

II. Ibid., p. 11 sq. 
Responce à l'antigaverston de Nogaret: A Monsieur d'Espernon, sur quatre anagrammes de son nom. Epernon fait alors composer une Lettre missive en forme de Response, à la replique de l'Antigaverston ${ }^{12}$. Le succès littéraire de la figure du couple Edouard II-Gaveston ${ }^{13}$ doit sans doute beaucoup à la cristallisation autour de ces personnages initiaux de toute l'imagerie postérieure de la cour des derniers Valois: le prince corrompu de l'automne de la Renaissance se réincarne, à rebours, dans le monarque médiéval décadent. Temps de l'histoire et temps du discours deviennent presque indissociables dans l'imaginaire, avec Edouard II comme signe d'Henri III ou Gaveston comme signe d'Epernon.

La figure de Gaveston rend ici précisément compte d'un débat sur le sujet du partage entre privé et public ou entre passion et raison, central dans le discours politique de la fin du XVI et du début du XVII e siècle et absolument essentiel pour la compréhension de la notion moderne émergente de corruption politique. Ce qui caractérise le mieux la relation du roi au favori est son abstraction de la sphère publique:

Gaverston [précise l'ironique épître dédicatoire de l'Histoire tragique et memorable de Pierre de Gaverston] ayant une fois occupé tous les cabinets des bonnes graces de son Roy, ou à mieux dire l'ayant infatué et ensorcelé, feist en sorte que autre que luy n'en pouvoit approcher faisant disgratier et esloigner de la Court tous les Princes qui y estoient auparavant bien venus. Si vous voulez nier que n'avez fait de mesme, vous serez seul qui deffendrez ceste negative, et quand il n'y auroit point de preuve, les parois et murailles des oratoires que vous avez faict faire au Louvre, afin que fussieez logé seul près du Roy, pour mieux enfiler vos affaires, le justifieront assez.

Autour d'une comparaison avec l'empereur romain Galien, L'Estrange Amitié décrit le roi Edouard II face à la ruine de son royaume:

Quel aveuglement je vous prie, quelle indignité, quelle cruauté, d'appauvrir tout un Royaume, de faire mourir de faim tant de gens,

I2. Voir M. Martin, "Queering/historiciser Gaveston", ainsi que G. Poirier, L'homosexualité dans l'imaginaire de la Renaissance et G. Ferguson, Queer (re)readings in the French Renaissance.

13. Et, ce, très rapidement, avec la tragédie de Marlowe. 
pour enrichir je ne sçay quels coquins, qui ne servent de rien au public $^{14}$.

Ce rapport exclusif est perçu comme un terrible danger pour la cour et le royaume, en ce qu'il altère profondément la «justice distributive que le roi doit employer à l'égard de sa noblesse " ${ }^{15}$, l'économie du don et du guerredon garante de l'ordre social féodal. Car, la réflexion sur la corruption au début de la période moderne, ne peut se faire que dans ces termes ${ }^{16}$, comme le souligne très justement Natalie Zemon Davies:

La France du XVI ${ }^{e}$ siècle mettait ainsi au cœur du débat un thème tout à fait central; au sein d'un monde de dons qui créaient des «amitiés» et des obligations de reconnaissance, où commençait la corruption? Dans la France de l'Ancien Régime, il n'existait pas même un terme spécifique pour désigner la corruption ${ }^{17}$. [...] Ainsi donc se servait-on simplement en France des mots «dons» ou "présents», et c'est en fonction du contexte ou du déroulement concret des événements qu'il fallait décider si le don était bon ou mauvais ${ }^{18}$.

Le détournement des ressources royales au profit d'un seul en priverait les réseaux traditionnels de redistribution aux clientèles des grands seigneurs. La captation des charges et des honneurs libère la jalousie de la noblesse, tout à fait envahissante dans la première moitié du XVII siècle ${ }^{19}$. Gaverston, tout comme Epernon, est coupable de faire naître ce sentiment chez les grands, juste sentiment puisqu'il doit garantir le bien public, s'il le faut par l'usage du droit de révolte. On lit, par exemple, cette adresse au duc d'Epernon dans la Replique à l'antigaverston:

Si eussiez esté si amateur du bien du service du Roy et du public, comme vous dictes estre, vous vous fussiez volontairement despouillé, de partie des charges et dignitez qu'avez ravies, sans permettre que le

I4. L'Estrange amitié d'Edouard Second, Roy d'Angleterre à l'endroit de Pierre de Gaverston, Gentilhomme de Gascogne, et quelle en fut l'yssue, p. 6.

I5. N. Le Roux, La faveur du roi, p. 628.

I6. Voir M. Génaux, "Les mots de la corruption».

17. Au contraire de l'Angleterre qui dispose très tôt du mot "bribe"; voir J. T. Noonan, Bribes.

I8. N. Z. Davis, Essai sur le don dans la France du XVIe siècle, p. 138.

19. Voir M. Bertaud, La jalousie dans la littérature au temps de Louis XIII. 
peuple fust ruiné par les gens de guerre qu'il convient d'assembler, pour les vous oster avec honte et ignominie. Par la jalousie qu'avez causé aux grands, on peut cognoistre, de combien il est pernicieux aux Roys de se laisser gouverner par gens de basse condition, et ne faire conte des Princes, Seigneurs, ny de la noblesse ${ }^{20}$.

La critique de la faute politique et sociale du moderne Gaverston se double d'une condamnation morale et d'un discours plus religieux. La juste jalousie des grands est la réponse mais en même temps presque le reflet du péché des favoris: l'envie. Celle-ci naît d'une comparaison déplacée du mignon à son roi qui rend compte de son désir de "passer pour supérieur à tous les autres, jouir de la gloire de la prééminence, recevoir les louanges qui reviennent au meilleur ${ }^{21}$ ». La recherche du pouvoir et des richesses donne lieu, dans les libelles, à l'élaboration de listes sur le modèle de la littérature carnavalesque; cependant, il ne s'agit plus d'y célébrer la variété, la richesse et la création mais de condamner la captation par un seul, la privation, la destruction. En faisant irruption dans l'histoire, le roi de carnaval est devenu un danger pour la société; l'inversion n'est plus promesse de vie mais péril social:

Tout ce que nous pouvons pour vostre Majesté Est, vous donner conseil en nostre conscience, Que vostre favory vous faciez Roy de France, Et soyez son amy tel qu'il vous a esté.

Vous changerez de Chance et serez fait semblable, Mis dessus puis dessoubs à l'orloge de sable, Qui remplit le dessus en le mettant dessoubs. Vous reprendrez l'Estat, les biens, et les richesses, Que vous avez perdu, par voz grandes largesses, Et sans necessité serez et vous et nous.

Dans ce noir carnaval, l'on reconnaît la main du diable; Epernon et Gaverston sont des sorciers qui ont utilisé leurs charmes pour s'approprier la personne du roi et rompre à travers lui le lien qui unit le

20. Replique à l'antigaverston, ou responce faicte à l'histoire de Gaverston par le duc d'Espernon, p. 21.

2I. C. Casagrande, S. Vecchio, Histoire des péchés capitaux au Moyen Age, p. 74. 
peuple à Dieu ${ }^{22}$. Leur marque est le mensonge. Les libelles dénoncent l'inversion si sensible dans les fausses louanges et la trompeuse renommée d'Epernon: ils tentent de remettre à l'endroit une langue pervertie par les thuriféraires des favoris. Les jeux anagrammatiques se comprennent dans cette logique: en réorganisant les lettres qui composent le nom usurpé du favori, ils disent la vérité sur la personne, sur sa nature d'"Archiharpie sangsuë de la France, esponge des finances, controuveur d'un million de monstrueux offices, grippe tout, happe don, ronge peuple, pipe Roy, sible court, et bref monstre odieux de ce Royaume tres-chrestien " ${ }^{23}$. La portée du «jeu», conçu comme activité ludique et comme mouvement ou contre-mouvement en réaction à la corruption des choses qui est aussi altération des mots et de leur sens, est ainsi résumée:

Combien que l'ingenieuse invention de tourner, retourner et changer les noms et surnoms des personnes, semble aux plus difficiles et rigoureux censeurs des sciences, comme un art sans, et que ce nouvel ou plustost antique lycophronisme ou façon d'anagrammes, soit comme une espee à deux tranchans, si est-ce qu'il y a de l'admiration de rencontrer à propos, ce qui est veritable au nom d'un personnage signalé, tel que le fameux successeur de son compatriote, Pierre de Gaverston, c'est ce grand et nouveau duc d'Espernon ${ }^{24}$.

Soit: Periure (Perjure, Parjure) de Nogaret pour Pierre de Gaverston, ou Jehan Loys de Nogareth pour «l'hydre huguenot à son ayse», Jehan Loys de Nogaret pour "en degast Roy n'a loy" ou encore duc d'Espernon pour "prend don d'escus». L'imaginaire de l'inversion qui recouvre les motifs aussi divers que la sodomie, l'efféminement, la sorcellerie ou encore l'ascension sociale illustre le refus de Dieu et de sa prodigalité : à travers la captation des faveurs du roi, se joue le renversement de la création divine, la stérilisation d'un ordre naturellement propice à l'homme. Et ce n'est qu'un certain usage de la parole de vérité qui permettra, par la force des mots et leur pouvoir de retournement, de combattre ce mouvement de corruption et de rétablir l'ordre moral, social et politique ancien. Cette

22. Sur le topos de la diabolisation d'Epernon, on lira la Guisiade de Pierre Matthieu et la documentation réunie sous le titre de "Pamphlets sur Henri III", dans Théâtre de la cruauté et récits sanglants en France (XVIe-XVII' siècle), p. 856-879.

23. Responce à l'antigaverston: A Monsieur d'Espernon, sur quatre anagrammes de son nom, p. 5.

24. Ibid. 
activité est d'abord celle de la chaire, celle des prédicateurs de la Ligue ${ }^{25}$, qui galvanisent les populations des villes ligueuses après l'exécution des Guise lors des états généraux de Blois et qui, peut-être, par la puissance de leur verbe, provoquent la chute du dernier des Valois sous les coups de couteau d'un moine illuminé, Jacques Clément ${ }^{26}$.

A la mort d'Henri III, personne ne crie "Vive le roi »; beaucoup de ses soutiens manquent au prétendant au trône, Henri de Navarre qui promet, pourtant rapidement, de maintenir la religion catholique et de se soumettre à un concile national. Le "Béarnais", pour ceux qui ne reconnaissent pas ses droits, est forcé de lever le siège de la capitale; ses maigres troupes résistent cependant aux puissantes armées de la Ligue et se maintiennent au nord de la Loire, remportant des victoires (le 21 septembre 1589 à Arques, le 14 mars à Ivry) certes peu décisives sur le plan militaire mais essentielles sur le plan symbolique puisqu'elles contribuent à l'émergence de l'image d'un « roi providentiel». En mai 1590, ses partisans encerclent à nouveau Paris; le siège de quatre mois est terrible: certes, la fièvre mystique anime toujours certains rebelles; mais les habitants souffrent, demandent "la paix ou le pain» et se dissocient de plus en plus des responsables de la Ligue et particulièrement des extrémistes du groupe des Seize (appelé ainsi en référence au nombre des quartiers de la ville). Du point de vue militaire, les ligueurs sont forcés d'en appeler aux Espagnols pour libérer Paris de l'emprise royale.

Cela modifie profondément les rapports de force au sein du mouvement: jusque-là, le duc de Mayenne, nommé lieutenant général du royaume à la mort de son frère Henri de Guise, jouissait d'une certaine latitude et parvenait à concilier les tendances nobiliaires et populaires du parti ligueur; mais les Espagnols veulent la désignation rapide d'un roi; leurs intérêts et ceux des princes lorrains divergent, bien évidemment, sur cette question. Ces dissensions culminent, le 15 novembre 1591, dans une tentative de coup de force des Seize dont le signal est donné avec l'assassinat du président de parlement Brisson et

25. Citons le plus forcené sans doute, Jean Boucher, curé de Saint-Benoît, recteur de la Sorbonne, instigateur du groupe des Seize, auteur prolixe d'ouvrages séditieux tels que L'Histoire tragique de Pierre de Gaverston, le De iusta abdicatione Henrici III, la Vie et faits notables de Henri de Valois, les Sermons de la simulée conversion et nullité de la prétendue absolution d'Henri de Bourbon, prince de Béarn ou encore l'Apologie pour Jehan Chastel.

26. Voir N. Le Roux, Un régicide au nom de Dieu, p. 160-181. 
de ses conseillers Larcher et Tardif, jugés trop mous. Mayenne revient à Paris et organise des purges, en faisant exécuter quatre ligueurs extrémistes, Ameline, Louchard, Aymonnot et Anroux. Les Seize ne lui pardonnent pas; il devient la cible de leurs attaques, en particulier dans l'un des plus grands libelles de l'époque, le Dialogue d'entre le maheustre et le manant ${ }^{27}$. C'est dans ce contexte tendu que les Espagnols exigent la tenue d'états généraux pour élire un roi catholique; Mayenne repousse l'échéance tant qu'il peut, mais il doit finalement se plier aux demandes insistantes de Philippe II : l'assemblée est solennellement ouverte à Paris le 26 janvier 1593.

Les ambitions des uns et des autres s'y étalent au grand jour: tous convoitent le royaume de France, Mayenne, le lieutenant de la Ligue, le jeune Charles de Guise, fils du martyr, mais aussi les ducs de Mercœur, de Savoie, de Lorraine. Cependant, Henri IV propose des pourparlers que l'assemblée accepte; pour contrecarrer la conférence de Suresnes ouverte le 29 avril, les ambassadeurs espagnols demandent, dès le 16 mai, le trône pour l'infante Isabelle, qui est la fille d'Élisabeth de France et la petite-fille d'Henri II ; les 20 et 21 juin, devant les réactions hostiles des députés à l'annonce de son projet de mariage avec l'archiduc Ernest de Habsbourg, ils proposent d'élire solidairement à la couronne l'infante et un prince français du choix des états, vraisemblablement le duc de Guise. L'opposition d'une partie de l'assemblée ravive les conflits traditionnels entre les états généraux et le Parlement, qui réaffirme, avec l'arrêt Le Maître du 28 juin, le caractère fondamental de la loi salique, qui exclut toute femme de la succession. Les députés sont contraints de se déclarer incompétents sur la question de l'élection du roi et se séparent en octobre. C'est cette "farce d'états» que moque un curieux manuscrit qui circule sous le manteau dès avril ou mai 1593: l'Abbregé et l'ame des estatz convoquez à Paris en l'an 1593, l'ébauche de ce qui devient, sous les presses royales, un an plus tard, La Vertu du catholicon puis la Satyre ménippée 28.

Tant le Dialogue d'entre le maheustre et le manant que la Satyre ménippée font référence à l'ordonnancement des états généraux en mettant en scène l'opposition de représentants allégoriques de la noblesse et du peuple pour le premier ou de caricatures d'orateurs réels pour le

27. L. Morin dit Cromé, Dialogue d'entre le maheustre et le manant.

28. Satyre Menippee de la Vertu du Catholicon d'Espagne. 
second; c'est que la tenue d'états est traditionnellement conçue comme le remède à la corruption du corps du royaume dans la vision organiciste de la société qui prévaut alors:

Et comment est-il aujourd'hui possible [s'interrogeait-on peu avant les premiers états de Blois de 1576] de maintenir cest Estat, pour pourvoir aux necessitez du Roy et du peuple, si ce n'est par ceste ancienne et sainte observance d'assembler les estats, par lesquels on pourra remedier à la corruption qui a tant gaigné sur la Religion et la Justice, qui sont les deux colomnes principales sur lesquelles est fondee la seureté de tous Royaumes, et aux bonnes loix qui ont du tout perdu leur vigueur. Pourtant pour asseurer ce Royaume d'une ruine qui autrement s'ensuivra bientost, il faut, tant pour la necessité qui nous y contraint que pour l'utilité qui nous invite à ce faire, tourner tous nos desseins et deliberations, remettre sus ceste coustume quasi oubliee, que le peuple puisse approcher librement de la personne de son Roy pour lui faire entendre ses plaintes, et le Roy vueille prendre advis et conseil de ses subjets quand il sera question des affaires qui concernent la conservation du Royaume, et de l'auctorité et puissance royale. Car il n'y a aujourd'huy esperance que la France puisse recouvrer sa splendeur, et esteindre toutes estincelles de partialité, si ce n'est par le conseil et les loix irrevocables desdits estats. [...] Autrement de brief en ce Royaume tempéré de trois sortes de bons gouvernemens la Monarchie se convertira en une Tyrannie extreme, l'Aristocratie, qui est quand peu de gens de bien commandent, en oligarchie où contre les loix peu de gens meschans, d'une reigle tortue et inegale, mesurent toutes choses sans aucun respect de justice à leur profit particulier. La Democratie qui est un estat populaire, en une Ochlocratie, qui est un estat turbulent et où toute meschanceté a plus de force que les loix et bonnes mœurs ${ }^{29}$.

Mais, depuis, était intervenue la tragédie des seconds états de Blois ensanglantés par les meurtres du duc Henri de Guise et de son frère

29. P. Vuallemand [Fr. Hotman], Remonstrances aus seigneurs gentilshommes et autres faisans profession de la Religion reformée en France, et tous aultres bons François desirans la conservation de ce Royaume, [repris dans] S. Goulart, Mémoires de l'estat de France sous Charles IX contenant les choses les plus notables, faictes et publiées tant par les catholiques que par ceux de la religion, depuis le troisième édit de pacification faict au mois d'Aoust 1570 jusques au règne de Henry troisieme, et reduits en trois volumes, chacuns desquels a un indice des principales matières y contenus. Troisiesme volume (nous soulignons). On reconnaîtra l'influence aristotélicienne (Politiques, 1292a-1319b) jusque dans le choix du vocabulaire. 
Louis, le cardinal; et puis, les actes des représentants des états sans roi de 1593 ne cessaient de marquer combien les députés étaient corrompus par les divers prétendants à la couronne; par-delà la reprise de la rhétorique habituelle de la critique des juges, du côté du Dialogue ${ }^{30}$, et de celle des mauvais prédicateurs, du côté de la Satyre ${ }^{31}$, dans la lignée des invectives religieuses pour le premier et avec la réappropriation, pour le second, d'une imagerie érudite et humaniste de la chute ${ }^{32}$ en même temps que d'un style grotesque inspiré de Rabelais mettant en scène la corruption des corps et des esprits ${ }^{33}$, c'est la même dénonciation du pouvoir de l'argent de part et d'autre, capable d'acheter les nobles précédemment alliés de la Ligue populaire défendue par l'auteur du Manant ou les différents acteurs des états de la Ligue susceptibles d'élire un roi, ce qui est inacceptable pour les rédacteurs de la Ménippée, soutiens du seul roi naturel, Henri IV. Une autre figure essentielle de la corruption est particulièrement présente dans ce dernier libelle: celle de l'ambassadeur ${ }^{34}$, et spécifiquement des émissaires de Philippe II, acteur principal de cette "corruption d'argent». Le lien entre la monnaie espagnole (le doublon) et l'ambiguïté est particulièrement fécond; s'appuyant sur un

30. L. Morin dit Cromé, Dialogue d'entre le maheustre et le manant, p. 94 sq: «ainsi Dieu s'est aydé, pour le fondement et commencement de la Ligue des catholiques de Paris, de feu M. Charles Hotoman, l'un des bourgeois d'icelle ville, homme trèsvertueux, de noble, bonne, ancienne et honneste famille, qui considerant la misere du temps, l'ambition des grands, la corruption de la justice et l'insolence du peuple, et surtout la perte de la Religion Catholique, Apostolique et Romaine, qui ne servoit que d'umbrage au peuple et de pretexte aux grands; et au contraire l'heresie supportee et la tyrannie ouverte, à ces occasions, meu de l'esprit de Dieu, il s'adressa à plusieurs docteurs, curez et predicateurs, pour sçavoir le moyen de s'y gouverner en seureté de conscience et pour le bien public».

3I. Par exemple dans les éloges ironiques constitués par les harangues de deux orateurs emblématiques de la Ligue, Claude d'Epinac, archevêque de Lyon et Guillaume Rose, évêque de Senlis, dans Satyre Menippee, p. 50-56 et 57-68.

32. Avec les références mythologiques récurrentes à Phaéton (p. 18, 19 et 174), Icare (p. 19) ou aux géants (p. 131).

33. C'est d'abord un pet que laisse échapper Madame de Bussy (p. 28); mais cela amorce une longue suite de dérèglements corporels qui rythment ces états (p. 38, 42, 49, 56, 67 sq., 74); et, dans le temps même où les corps se détraquent, les discours se délitent (l'un des orateurs, Rose, passe pour fou: p. 57-68).

34. Voir M. Martin, "Ambassadeurs, espions et comploteurs espagnols et “espagnolisés” dans les libelles de la Ligue (1584-1598)». Comme le résume N. Z. Davis: "La diplomatie était un autre réseau du don concerné par le problème de la corruption" (Essai sur le don dans la France du XVI e siècle, p. 238). 
jeu de mots facile, mais aussi du coup tout à fait accessible et parlant au plus grand nombre, - le doublon rend double ${ }^{35}$-, il permet de renvoyer en même temps à l'image traditionnelle du Français comme franc; être payé d'argent espagnol, c'est s'éloigner dangereusement de la naïveté et de la simplicité française pour se rapprocher de l'hypocrisie espagnole. Le traître "espagnolisé» tient un discours trompeur où les intérêts financiers (sinon politiques) se dissimulent sous le prétexte de religion. La Satyre ménippée entend donc substituer le texte royaliste à la mauvaise ou à la fausse monnaie. Mais la satire n'est qu'une étape préliminaire dans la formation d'un "texte-valeur", signe de la gloire et de la sagesse du roi; les rédacteurs du libelle sont conscients de ne pas servir à la restauration du système ancien du pouvoir et de la société traditionnelle du don - même s'ils s'en servent comme modèles, comme cautions -, mais à l'instauration d'un pouvoir nouveau et sans partage.

Pour conclure après ce trop rapide aperçu historique sur la période de la Ligue, la notion de "corruption" permet de bien saisir les évolutions de la production "pamphlétaire" de ces années 1584-1598. La reprise de la critique de la corruption dans les libelles rend d'abord compte de l'ambiguïté du positionnement politico-religieux de la Ligue à ses origines (nous avons vu comment avaient successivement paru deux versions du manifeste du mouvement, l'une privilégiant d'abord la dimension politico-financière de la corruption, l'autre tentant après coup de se raccorder à une tradition religieuse); cependant, ensuite, dans un contexte où les favoris du roi Henri III et singulièrement Epernon cristallisent les accusations d'accaparement des biens royaux et de sorcellerie, la corruption devient un thème unificateur qui explique le succès de la propagande ligueuse autour de 1588 sans que les défenses royales ne parviennent à retourner cette accusation contre les leaders charismatiques de la Ligue; enfin, ce sont les dissensions internes au mouvement qui débouchent, après 1592 , sur des accusations d'ambition personnelle ou de subornation aux intérêts étrangers contre les nobles et sur la dénonciation des turpitudes de la populace; la nouvelle propagande royale organisée autour de la figure du roi providentiel Henri IV récupère ces discours ligueurs contre la corruption ligueuse tout en reconnaissant en eux la cause

35. "Les François simplets paravant, / Sont par doublons devenuz doubles: / Et les doublons tournez en vent, / Ou bien en cuyvre, et rouges doubles» (p. 116, voir aussi p. 137). 
première des conflits armés, des misères de la guerre et de la corruption du royaume de France. Qu'en est-il de ce discours si versatile de la corruption à l'issue de cette période? Henri IV est rapidement perçu comme ingrat envers ceux qui l'ont servi (et Dieu, qu'il a servi ?) et favorable à ses anciens ennemis plus utiles politiquement: dans la Confession du sieur de Sancy, dans les Avantures du baron de Faneste, D'Aubigné est le témoin privilégié de ce nouvel effondrement des valeurs. Et ce n'est donc pas seulement la période des guerres de la Ligue mais bien la totalité des règnes d'Henri III et surtout d'Henri IV que le meilleur témoin de ces échanges pamphlétaires, Pierre de L'Estoile, avec qui nous avons ouvert et avec qui nous refermons ce panorama, désignera comme un «temps du tout corrompu»:

Force maladies à Paris en ce mois; mortalité de petits enfans par les petites véroles qui règnent. Le curé de Saint-Nicolas-des-Champs dit à un mien ami qu'il en avoit enterré trois cens en sa paroisse, depuis le commencement de l'année jusques à ce jour. Hors Paris, encore pis. On fait compte à Chartres de sept à huit cens enfans au moins: car autres en mettent jusques à douze cens emportés de ladite maladie. A Lion, de plus de trois mille; et ainsi aux autres villes et endroits de la France. Ce mal aiant couru partout, et courant encores aujhourdui, ravage comme un torrent une infinité de ces petites ames innocentes; quelques grands en sont mesme attaints, et en passent le pas; mais peu, et plus de femmes que d'hommes. Beaucoup de fiebvres continues, mesme de pourprées, signe de grande corruption (comme aussi la disposition de l'air de ceste saison estoit maligne, remplie de tonnerres, orages, pluies impétueuses et tempestes, symbolizante aux humeurs du siècle), en font desloger grand nombre de tous aages, sexes et qualités. Les courantes, les apoplexies, et diverses sortes de morts subites et estranges en tuent d'autre costé tout plain et estonnent le peuple, qui pour cela n'en amende guères.

Quant aux affaires publiques, il ne se parle que d'imposts, enchères d'estats, nouveaux édits et ordonnances onéreuses au peuple, à qui oster le bien c'est lui oster la vie: estant chose véritable (tesmoin Tacite au quatrième livre de ses Annales) que les lois abondent et multiplient en un Estat lorsqu'il est plus corrompu, et que l'avarice (que Cicéron, au deuxième livre de ses Offices, apèle très infâme, sur tout ès princes et 
magistrats) a le crédit et la vogue, comme nous le voions en ce misérable siècle ${ }^{36}$.

Certes, L'Estoile souligne, comme les libelles ou les rumeurs qu'il compile, et avec une grande variété des approches entre le ressenti naïf du petit peuple et la vision religieuse, la particulière corruption de la justice ${ }^{37}$, de la police ${ }^{38}$, de la cour ${ }^{39}$, des mours sexuelles ${ }^{40}$ et du sentiment religieux ${ }^{41}$ ou le rôle singulier de l'argent ${ }^{42}$ dans ce mouvement général; mais, à la manière de Montaigne ${ }^{43}$, il critique les "livres ineptes qui ressentent la corruption du siecle " ${ }^{44}$ et reconnaît dans la moquerie ${ }^{45}$ et la médisance ${ }^{46}$ les signes de la plus grande corruption possible. Comme le formule si bien Gilbert Schrenck, «l'activité pamphlétaire est le symptôme de la déchéance spirituelle" ${ }^{47}$. Le libelle aux XVI ${ }^{\mathrm{e}}$ et XVII ${ }^{\mathrm{e}}$ siècles se situe donc au centre de la réflexion sur les pouvoirs de la langue: s'il prétend rétablir l'ordre en dénonçant la corruption, il se révèle souvent lui-même corrupteur. Langue, où vas-tu? Renverser la cité puis redresser la cité?

Martial Martin

Université de Reims

36. P. de L'Estoile, Registre Journal, seconde partie du premier tome, p. 529 (nous soulignons).

37. Première partie du premier tome, p. 138; seconde partie du premier tome, p. 412 , p. 443 , p. 513 , p. $578-579$ ou p. 661.

38. Seconde partie du premier tome, p. 661 sq.

39. Première partie du premier tome, p. 91.

40. Seconde partie du premier tome, p. 661.

4I. Ibid., p. 436 ou 636 sq.

42. Seconde partie du premier tome, p. 628, p. 644 ou p. 661.

43. Montaigne, Essais, III, 9, p. 990 sq: "l'escrivaillerie semble estre quelque symptome d'un siecle desbordé».

44. Pierre de L'Estoile, Registre Journal, Seconde partie du premier tome, p. 559.

45. Première partie du premier tome, p. 98: «La corruption de ce temps est[ait] telle que les farceurs, bouffons, p... et mignons avoient tout le crédit».

46. Première partie du premier tome, p. 141.

47. G. Schrenck, "Jeu et théorie du pamphlet dans le Journal du règne de Henri III (1574-1589)», p. 74 sq. 


\section{BIBLIOGRAPHIE}

Textes

Cromé, Louis Morin dit, Dialogue d'entre le maheustre et le manant [1593-1594], éd. par Peter Ascoli, Genève, Droz, 1977.

Declaration des causes qui ont meu Monseigneur le Cardinal de Bourbon et les Pairs, Princes, Seigneurs, Villes et Communautez catholiques de ce Royaume de s'opposer par armes à ceux qui veulent subvertir la Religion, Leyde, T. Basson, 1585.

Estoile, Pierre de l', Registre Journal, Nouvelle Collection des mémoires pour servir à l'histoire de France, éd. par Joseph-François Michaud et Jean-Joseph-François Poujoulat, Paris, Chez l'éditeur du commentaire analytique du Code civil, 1837.

L'Estrange amitié d'Edouard Second, Roy d'Angleterre à l'endroit de Pierre de Gaverston, Gentilhomme de Gascogne, et quelle en fut l'yssue, [s. l.], [s. n.], [s. d.].

Matthieu, Pierre, La Guisiade, éd. par Louis Lobbes, Genève, Droz, 1990.

Replique à l'antigaverston, ou responce faicte à l'histoire de Gaverston par le duc d'Espernon, [s. 1.], [s. n.], 1588.

Responce à l'antigaverston: A Monsieur d'Espernon, sur quatre anagrammes de son nom, [s. l.], [s. n.], 1588.

Satyre Menippee de la Vertu du Catholicon d'Espagne [1593-1595], éd. Martial Martin, Paris, Honoré Champion, 2007.

Vuallemand, Pieter, [François Hotman], Remonstrances aus seigneurs gentilshommes et autres faisans profession de la Religion reformée en France, et tous aultres bons François desirans la conservation de ce Royaume, Bâle, 1574, [repris dans] Simon Goulart, Mémoires de l'estat de France sous Charles IX contenant les choses les plus notables, faictes et publiées tant par les catholiques que par ceux de la religion, 
depuis le troisième édit de pacification faict au mois d'Aoust 1570 jusques au règne de Henry troisieme, et reduits en trois volumes, chacuns desquels a un indice des principales matières y contenus. Troisiesme volume, H. Wolf, Meidelbourd, 1578.

\section{Travaux}

Bertaud, Madeleine, La jalousie dans la littérature au temps de Louis XIII, Genève, Droz, 1981.

Biet, Christian (éd.), Théâtre de la cruauté et récits sanglants en France (XVI'-XVII ${ }^{e}$ siècle), Paris, Robert Laffont, 2006.

Casagrande, Carla, et Vecchio, Silvana, Histoire des péchés capitaux au Moyen Age, Paris, Aubier, 2003.

Constant, Jean-Marie, La Ligue, Paris, Fayard, 1996.

Crouzet, Denis, Les guerriers de Dieu. La violence au temps des troubles de religion, vers 1525-vers 1610, Seyssel, Champ Vallon, 1990.

Davis, Nathalie Zemon, Essai sur le don dans la France du XVIe siècle, Paris, Seuil, 2003.

Ferguson, Gary, Queer (re)readings in the French Renaissance. Homosexuality, Gender, Culture, Aldershot, Ashgate, 2008.

Génaux, Maryvonne, "Les mots de la corruption. La déviance publique dans les dictionnaires d'Ancien Régime", Les Annales. Histoire, économie et société, 21/4 (2002), p. 513-530.

Jounnna, Arlette, Boucher, Jacqueline, Biloghi, Dominique, Histoire et dictionnaire des guerres de religion, Paris, Robert Laffont, 1998.

Le Roux, Nicolas, La faveur du roi. Mignons et courtisans au temps des derniers Valois (vers 1547-vers 1589), Seyssel, Champ Vallon, 2001.

—, Un régicide au nom de Dieu. L'assassinat d'Henri III, Paris, Gallimard, 2006.

Martin, Martial, "Queering/historiciser Gaveston: les libelles de la Ligue et la Fronde", in Queer Strategies, éd. par Pierre Zobermann, Paris, L'Harmattan, 2008, t. 1, p. 103-116.

—, "Ambassadeurs, espions et comploteurs espagnols et "espagnolisés" dans les libelles de la Ligue (1584-1598)", in Ambassadeurs, apprentis espions et maîtres comploteurs. Les systèmes de renseignements à l'époque moderne, éd. par Béatrice Pérez, Annie Molinié, Paris, PUPS, 2010, p. 243-258. 
Noonan, John T., Bribes, New York, Macmillan Publishing Company, 1984.

PoIrier, Guy, L'homosexualité dans l'imaginaire de la Renaissance, Paris, H. Champion, 1996.

Schrenck, Gilbert, "Jeu et théorie du pamphlet dans le Journal du règne de Henri III (1574-1589) de Pierre de l'Estoile», Traditions polémiques, Cahiers V. L. Saulnier, 2 (1984), p. 69-79. 
